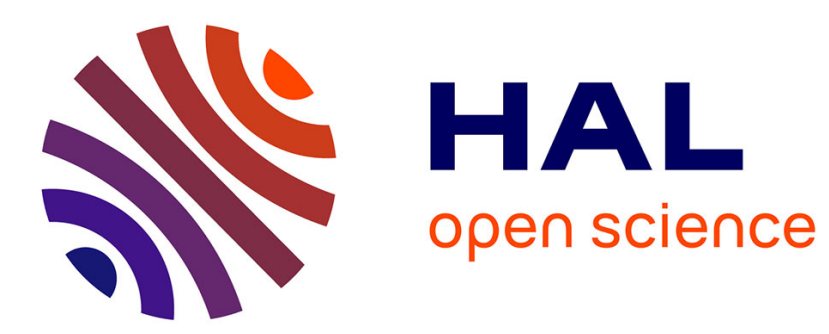

\title{
21. Étude de films minces d'alliages magnétiques dilués (CuFe) par effet tunnel
}

L. Dumoulin, E. Guyon, P. Nédellec

\section{To cite this version:}

L. Dumoulin, E. Guyon, P. Nédellec. 21. Étude de films minces d'alliages magnétiques dilués $(\mathrm{CuFe})$ par effet tunnel. Revue de Physique Appliquée, 1970, 5 (6), pp.907-907. 10.1051/rphysap:0197000506090701 . jpa-00243491

HAL Id: jpa-00243491

https://hal.science/jpa-00243491

Submitted on 1 Jan 1970

HAL is a multi-disciplinary open access archive for the deposit and dissemination of scientific research documents, whether they are published or not. The documents may come from teaching and research institutions in France or abroad, or from public or private research centers.
L'archive ouverte pluridisciplinaire HAL, est destinée au dépôt et à la diffusion de documents scientifiques de niveau recherche, publiés ou non, émanant des établissements d'enseignement et de recherche français ou étrangers, des laboratoires publics ou privés. 


\title{
21. ÉTUDE DE FILMS MINCES D’ALLIAGES MAGNÉTIQUES DILUÉS (CuFe) PAR EFFET TUNNEL
}

\author{
L. DUMOULIN, E. GUYON et P. NÉDELLEC
}

Laboratoire de Physique des Solides, Faculté des Sciences d'Orsay, Orsay

Les films sont obtenus dans un évaporateur ultra vide par bombardement électronique. Le comportement résistif des films de $\mathbf{C u F e}$ correspond, avec un bon accord quantitatif, au comportement «Kondo » du matériau massif $(\mathbf{C u F e} \sim 300 \mathrm{ppm})$. Nous avons noté l'absence systématique d'une structure dépendant de la concentration sur la mesure de la conductance de films $\mathbf{C u F e}$ (avec une précision relative de $10^{-3}$ à $10^{-4}$ ) [1]. (Ce résultat peut être comparé à l'absence des structures de phonons pour la caractéristique d'une jonction de $\mathrm{Pb}$ normal par rapport au cas où le $\mathrm{Pb}$ serait supraconducteur). Cependant en utilisant un effet de proximité entre le film de $\mathbf{C u F e}$ et un film de $\mathrm{Pb}$ supraconducteur, il est possible d'observer un affaiblissement des propriétés supraconductrices (température critique plus basse, comportement sans bande interdite plus important, ...) par rapport à un film de $\mathrm{Cu}$ pur étudié dans les mêmes conditions [2]. Une estimation numérique indique que l'effet des impuretés magnétiques diluées est renforcé par rapport au comportement prédit par le modèle d'AbrikoscvGorkov. Un tel comportement a été prédit pour un alliage dilué supraconducteur homogène quand $T_{K}$ est de l'ordre de la température critique (ici pour $\mathbf{C u F e}$
$\left.T_{K} \sim 10^{\circ} \mathrm{K}\right)$. Notons cependant que nous n'avons pas observé de structures caractéristiques lors de l'étude du bilame soit du côté $\mathrm{CuFe}$, soit du côté $\mathrm{Pb}$ qui auraient pu être reliées à l'existence d'états liés étroits dans la bande d'énergie interdite.

Nous présentons également une analyse quantitative des bilames par émission ionique secondaire. On montre que les effets de diffusion entre les deux constituants $\mathrm{Pb}$ et $\mathrm{Cu}$ sont négligeables et que la concentration du $\mathrm{Fe}$ est constante à l'intérieur du film de $\mathrm{CuFe}$. Nous n'avons pas trouvé de $\mathrm{O}_{2}$ à l'interface $\mathrm{Pb}-\mathrm{Cu}$ ce qui permet de conclure à une «interface propre ». Ces mesures indiquent que nous sommes dans la limite des expériences de proximité bien contrôlées.

Nous remercions M. Slodzian pour d'utiles discussions et $M$. Deschamps pour le travail effectué sur l'appareil d'émission ionique secondaire.

\section{Bibliographie}

[1] Dumoulin (L.), Guyon (E.), Rochlin (G. I.), Sol. State communications, $1970, \mathbf{8}, 287$.

[2] Dumoulin (L.), Guyon (E.), Nédellec (P.), Sol. State communications, $1970, \mathbf{8}, 885$. 